\title{
GMR
}

\section{Chemokine receptor CXCR4 and its ligand CXCL12 expressions and clinical significance in bladder cancer}

\author{
D.L. Yang, M.M. Xin, J.S. Wang, H.Y. Xu, Q. Huo, Z.R. Tang and H.F. Wang \\ Department of Urology, The Second Affiliated Hospital of Kunming Medical University, \\ Yunnan Institute of Urology, Kunming, China \\ Corresponding author: H.F. Wang \\ E-mail: highphone@126.com \\ Genet. Mol. Res. 14 (4): 17699-17707 (2015) \\ Received August 14, 2015 \\ Accepted October 7, 2015 \\ Published December 21, 2015 \\ DOI http://dx.doi.org/10.4238/2015.December.21.43
}

ABSTRACT. It is well known that chemokine receptors and their ligands play important roles in mediating the invasion and metastasis of malignant tumors. This aim of this study was to investigate the expression and clinical significance of chemokine receptor CXCR4 and its ligand CXCL12 in bladder tumor tissues. Cancerous and adjacent normal bladder tissues were collected from 42 patients. The expressions of CXCR4 and CXCL12 proteins were then detected by immunohistochemistry, and the expressions of CXCR4 and CXCL12 mRNAs were detected by RT-PCR. Bladder cancer tissues showed higher positive expressions of CXCR4 and CXCL12 than those in normal bladder mucosal tissues $(z=7.332$, 6.758, $\mathrm{P}<0.001$ ). Positive expressions of CXCR4 and CXCL12 were related to the differentiation degree and invasive depth of cancer tissues $(z=2.598-4.594$, $P<0.05)$, but not to patient gender or age $(z=0.273$ $0.554, \mathrm{P}>0.05)$. The expression of CXCR4 was positively correlated to CXCL12 expression in bladder cancer tissues $(r=0.661, \mathrm{P}<0.05)$. RTPCR revealed that CXCR4 and CXCL12 mRNAs were not expressed in normal tissues. Moreover, with increased depth of invasion, CXCR4 and CXCL12 mRNA expressions gradually increased in bladder cancer tissues 
and showed significant intergroup differences $(F=56.642,67.928, \mathrm{P}<$ 0.01). Taken together, these results indicate that the chemokine receptor CXCR4 and its ligand CXCL12 play important roles in the occurrence and development of bladder cancer.

Key words: Bladder cancer; Bladder tumor tissue; CXCR4; SDF-1; CXCL12

\section{INTRODUCTION}

Bladder cancer is a major urogenital cancer, and is characterized by high progression and recurrence rates. In China, bladder tumors are the most common among all urogenital cancer tumor types and their incidence is on the rise (Raghavan, 2012). According to numerous studies on the invasive mechanisms of tumor metastasis, chemokine receptors and their ligands play important roles in mediating the invasion and metastasis of malignant tumors (Dobner et al., 2012; Domanska et al., 2013). In the current study, we targeted the chemokine receptor CXCR4 and its ligand CXCL12, and investigated their protein and gene expressions using the immunohistochemistry (IHC) methods and reverse-transcriptase polymerase chain reaction (RT-PCR), respectively, in cancerous and normal bladder tissues, and analyzed their clinical significance.

\section{MATERIAL AND METHODS}

\section{Specimens}

Forty-two patients (35 males and 8 females) aged 42-86 years (median 67 years) undergoing operation between July 2010 and June 2011 were enrolled in this study. Postoperatively, all patients were pathologically diagnosed with bladder cancer. No patient received preoperative radiotherapy or chemotherapy. Additionally, normal mucosal bladder tissues (pathological sections confirmed no proliferative or tumor-induced changes) $5 \mathrm{~cm}$ above cancer tissues were collected from each patient as controls. Each specimen was processed in duplicate, one was fixed in $4 \%$ formaldehyde and one immediately stored at $-80^{\circ} \mathrm{C}$ until further analysis.

\section{Reagents}

Rabbit anti-human CXCR4 and anti-SDF-1 (CXCL12) monoclonal antibodies were purchased from Abcam (Cambridge, MA, USA). Astreptavidin peroxidase (S-P) immunohistochemical staining kit and DAB color kit were purchased from Fuzhou Maxim Biotech (Fujian, China). TRIzol Reagent for RNA extraction and the M-MLV First-Strand Synthesis Kit for RT-PCR were purchased from Invitrogen (Carlsbad, CA, USA). A Premix Taq kit for amplification of target genes with RT-PCR and DNA markers were purchased from TaKaRa Biotechnology (Dalian, China). CXCR4, CXCL12, and GAPDH (internal control) primers were synthesized by Shanghai Invitrogen (China). All other reagents were purchased from Sigma (US) or Amresco (US), or were produced in China at analytical grade.

\section{CXCR4 and CXCL12 protein expression in cancerous and normal bladder tissues detected by the IHC S-P method}

The resected bladder cancer tissues and normal bladder tissues were aseptically collected. 
After fixation with $4 \%$ paraformaldehyde and paraffin embedding, serial sections approximately 4 $\mu \mathrm{m}$ thick were obtained and mounted onto glass slides, followed by air drying and heating in an oven at $65^{\circ} \mathrm{C}$ for $2 \mathrm{~h}$. Then, after dewaxing by xylene and hydration by gradient ethanol, $0.3 \%$ $\mathrm{H}_{2} \mathrm{O}_{2}$ ( $\left.\mathrm{v} / \mathrm{v}\right)$ was used to inhibit endogenous peroxidase. The sections were then immersed in $10 \%$ citrate antigen repair solution and heated in a pressure cooker for $90 \mathrm{~s}$. After cooling, the tissue antigens were repaired, and $3 \% \mathrm{H}_{2} \mathrm{O}_{2}(\mathrm{v} / \mathrm{v})$ was used to inhibit endogenous peroxidase. The sections were then blocked with normal goat serum, and the anti-CXCR4 (working diluted $1: 100)$ and anti-SDF-1 (1:50) antibodies were separately added, and refrigerated overnight at $4^{\circ} \mathrm{C}$. The next day, the sections were sufficiently washed (5 $\min \times 3)$ with phosphate buffer solution (PBS), dripped with a biotin-conjugated secondary antibody working solution, and incubated at $37^{\circ} \mathrm{C}$ for $15 \mathrm{~min}$. The sections were then washed ( $\left.5 \mathrm{~min} \times 3\right)$ with PBS, dripped with a streptomycin$\mathrm{H}_{2} \mathrm{O}_{2}$ working solution, and incubated at room temperature for $10 \mathrm{~min}$. Then after washing (5 min $x 3$ ) with PBS, the sections were colored with a freshly prepared DAB color solution according to manufacturer instructions, re-dyed and clarified with hematoxylin, and sealed with a neutral resin before observation under light microscopy. In negative controls, PBS was used in lieu of the primary antibody.

\section{CXCR4 and CXCL12 mRNA expression detected by RT-PCR}

Approximately $100 \mathrm{mg}$ of the cold-stored specimens was collected and ground to a powder. RNA was extracted by adding $1 \mathrm{~mL}$ TRIzol Reagent. Absorptions of RNA at 260 and 280 $\mathrm{nm}$ were determined by ultraviolet spectrometry, and RNA concentration and purity were calculated. cDNA was synthesized by reverse transcription according to the M-MLV First-Strand Synthesis Kit instructions. The cDNA was then used as a template for PCR amplification of CXCR4 and CXCL12 transcripts, and GAPDH served as the internal control. PCR primers were as follows for CXCR4: 5'-CTCCTCTTTGTCATCACGCTTCC-3' (forward), 5'-GGATGAGGACACTGCTGTAGAG-3' (reverse); CXCR12: 5'-CTCAACACTCCAAACTGTGCCC-3' (forward), 5'-CTCCAGGTACTCCTGAATCCAC-3' (reverse); and GAPDH: 5'-AGAAGGCTGGGGCTCATTTG-3' (forward), 5'-AGGGGCCATCCACAGT CTTC-3' (reverse). Amplified fragment lengths for CXCR4, CXCL12, and GAPDH were 126, 104, and $258 \mathrm{bp}$, respectively. Reactions included pre-denaturation at $94^{\circ} \mathrm{C}$ for $3 \mathrm{~min}$; followed by 30 cycles of denaturation at $94^{\circ} \mathrm{C}$ for $30 \mathrm{~s}$, renaturation at $55^{\circ} \mathrm{C}$ for $30 \mathrm{~s}$, and extension at $72^{\circ} \mathrm{C}$ for $60 \mathrm{~s}$; and a final extension at $72^{\circ} \mathrm{C}$ for $5 \mathrm{~min}$. After amplification, $5 \mu \mathrm{L}$ of the products was collected for electrophoresis on a $1.5 \%$ agarose gel (containing ethidium bromide) followed by visualization and quantification with a gel imaging system.

\section{CXCR4 and CXCL12 protein expression detected by western blot}

For cell fractionation, approximately $100 \mathrm{mg}$ of bladder tissues was homogenized in liquid nitrogen and the homogenate was extracted in $2 \mathrm{~mL}$ homogenization buffer. The crude extract was cleared of debris by centrifugation $\left(4000 \mathrm{~g}, 40 \mathrm{~min}, 4^{\circ} \mathrm{C}\right)$. The microsomal fraction was separated from the soluble fraction by ultracentrifugation $\left(100,000 \mathrm{~g}, 30 \mathrm{~min}, 4^{\circ} \mathrm{C}\right)$. The pellet was washed three times in homogenization buffer supplemented with $0.05 \%$ Triton X-100 and resuspended in $50 \mu \mathrm{L}$ SDS-PAGE sample buffer. The soluble fraction was mixed with SDS-PAGE sample buffer (ratio: 2:1 v/v). For SDS-PAGE, $18 \mu \mathrm{L}$ soluble fraction and $10 \mu \mathrm{L}$ microsomal fraction were loaded. CXCR4 and CXCL12 protein were detected in $100 \mu \mathrm{g}$ protein, according to procedures described by Calogero et al. (2001) and Baron et al. (2006), and GAPDH was used as a loading control. 


\section{Examination of IHC results}

After positive staining, CXCR4 and CXCL12 appeared as light brown granules in the membrane and/or cytoplasm. Three fields of view obtained from a high power objective (400X) were selected for each section for analysis. A similar number of total cells was counted for each sample, and according to the percentage of positive cells, the IHC results were classified into: negative (-) $(5 \%)$, weak positive $(+)(5-25 \%)$, medium positive $(++)(25-50 \%)$, and strong positive $(+++)(\geq 50 \%)$.

\section{Analysis of RT-PCR results}

Band optical densities (BODs) of amplified CXCR4 and CXCL12 fragments obtained from the gel electrophoresis imaging system, were compared with the corresponding GAPDH BODs as the internal control. The Image J software (developed by US National Institutes of Health) was used for optical density analysis.

\section{Statistical analysis}

Data were statistically processed with SPSS 17.0 (SPSS Inc., Chicago, IL, USA). Grade data were compared by nonparametric statistical rank and Mann-Whitney tests. Correlations were examined by Spearman tests. Measurements were compared by ANOVA, completely and randomly designed variance analysis. Inspection level is $\alpha=0.05$. $P<0.05$ was considered to be significant.

\section{RESULTS}

\section{Protein expression of CXCR4 and CXCL12 in bladder tumor tissues and normal bladder tissues}

Positive expressions of CXCR4 and CXCL12 in bladder cancer tissue were significantly higher than those in normal bladder tissue $(z=7.332-6.758, P<0.001)$ (summarized in Figure 1 and Table 1).

\section{Clinical significance of CXCR4 and CXCL12 protein expressions in bladder tumor tissues}

A statistical association between the protein expression of CXCR4/CXCL12 and bladder cancer was observed, while only trends towards statistical associations were observed between CXCR4/CXCL12 protein expression and the degree of differentiation $(z=2.828, P<0.05)$ and depth of invasion ( $z=4.594, \mathrm{P}<0.01$ ) (Table 2). CXCR4/CXCL12 protein expression was found to be strongly associated with the progression and invasion of bladder cancer. No statistical associations between CXCR4/CXCL12 expression levels and the other clinical parameters analyzed were found (summarized in Table 2).

\section{Correlation between the degree of expression of CXCR4 and CXCL12 in bladder tumor tissues}

The expressions of CXCR4 and CXCL12 in bladder cancer tissue were positively correlated $(r=0.661, \mathrm{P}<0.05)$ (summarized in Table 3). 

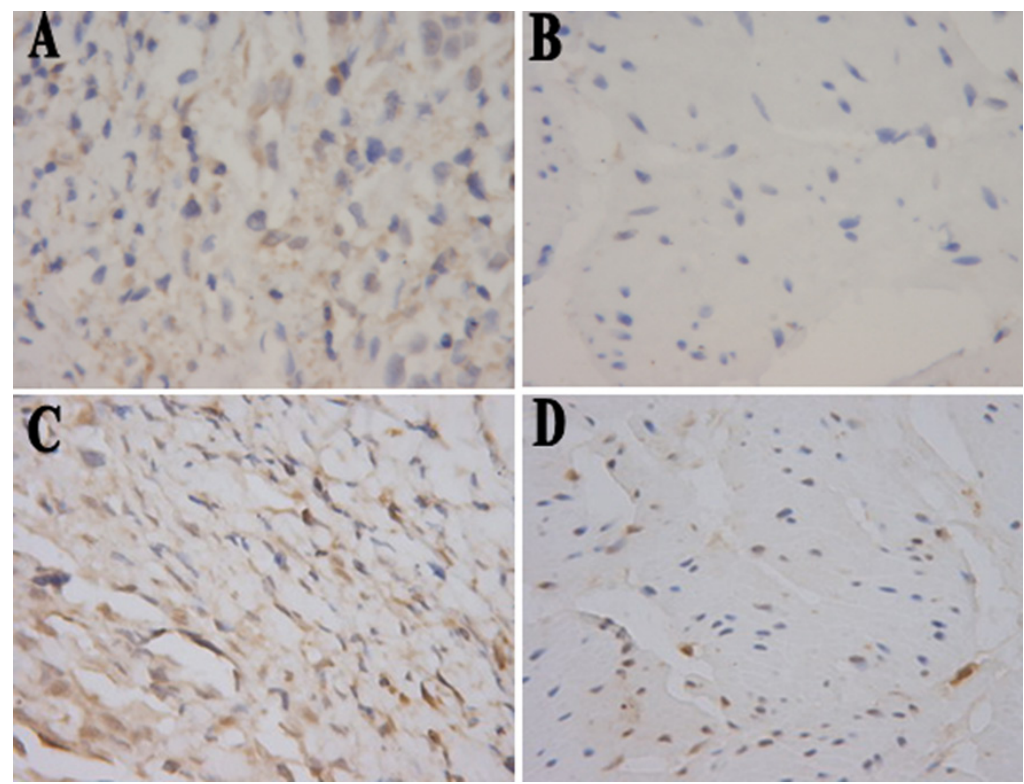

Figure 1. Immunohistochemical staining for CXCR4 and CXCL12. A. Expression of CXCR4 in bladder tumor tissues (S-P method, 400X). B. Normal bladder tissues do not express CXCR4 (S-P method, 400X). C. Expression of CXCL12 in bladder tumor tissues (S-P method, 400X). D. Normal bladder tissues do not express CXCL12 (S-P method, 400X).

Table 1. Comparison of CXCR4 and CXCL12 expression between normal bladder tissues and bladder cancer tissues (cases).

\begin{tabular}{|c|c|c|c|c|c|c|c|c|c|}
\hline \multirow[t]{2}{*}{ Group } & \multirow[t]{2}{*}{$\mathrm{N}$} & \multicolumn{4}{|c|}{ CXCR4 } & \multicolumn{4}{|c|}{ CXCL12 } \\
\hline & & - & + & ++ & +++ & - & + & ++ & +++ \\
\hline Bladder tumor tissues & 42 & 36 & 5 & 1 & 0 & 35 & 5 & 2 & 0 \\
\hline Normal bladder tissues & 42 & 3 & 10 & 11 & 18 & 5 & 9 & 12 & 16 \\
\hline
\end{tabular}

Table 2. Relationship between CXCR4/CXCL12 protein expressions and clinical parameters (cases).

\begin{tabular}{|c|c|c|c|c|c|c|c|c|c|c|c|c|c|}
\hline \multirow[t]{2}{*}{ Clinical parameter } & \multirow[t]{2}{*}{$\mathrm{N}$} & \multicolumn{6}{|c|}{ CXCR4 } & \multicolumn{6}{|c|}{ CXCL12 } \\
\hline & & - & + & ++ & +++ & $z$ & $P$ value & - & + & ++ & +++ & $z$ & $P$ value \\
\hline \multicolumn{14}{|l|}{ Gender } \\
\hline Male & 34 & 3 & 7 & 9 & 15 & 0.272 & $>0.05$ & 5 & 6 & 11 & 12 & 0.554 & $>0.05$ \\
\hline Female & 8 & 0 & 3 & 2 & 3 & & & 0 & 3 & 1 & 4 & & \\
\hline \multicolumn{14}{|l|}{ Age (years) } \\
\hline$\leq 67$ & 21 & 1 & 6 & 4 & 10 & 0.333 & $>0.05$ & 3 & 4 & 7 & 7 & 0.461 & $>0.05$ \\
\hline$>67$ & 21 & 2 & 4 & 7 & 8 & & & 2 & 5 & 5 & 9 & & \\
\hline \multicolumn{14}{|l|}{ Depth of invasion } \\
\hline $\mathrm{Ta}+\mathrm{T} 1$ & 27 & 3 & 10 & 8 & 6 & 3.798 & $<0.01$ & 5 & 9 & 10 & 3 & 4.594 & $<0.01$ \\
\hline $\mathrm{T} 2+\mathrm{T} 3$ & 15 & 0 & 0 & 3 & 12 & & & 0 & 0 & 2 & 13 & & \\
\hline \multicolumn{14}{|c|}{ Degree of differentiation } \\
\hline Medium to high & 19 & 2 & 7 & 6 & 4 & 2.598 & $<0.05$ & 3 & 6 & 8 & 2 & 2.821 & $<0.05$ \\
\hline Low & 23 & 1 & 3 & 5 & 14 & & & 2 & 3 & 4 & 14 & & \\
\hline
\end{tabular}


Table 3. Correlation between CXCR4 and CXCL12 expressions in bladder tumor tissues (cases).

\begin{tabular}{lllrrr}
\hline CXCR4 & & & CXCL12 & & Total \\
\cline { 2 - 5 } & - & + & ++ & +++ & \\
\hline- & 2 & 1 & 0 & 0 & 3 \\
+ & 1 & 3 & 6 & 0 & 10 \\
++ & 2 & 4 & 2 & 3 & 11 \\
+++ & 0 & 1 & 4 & 13 & 18 \\
Total & 5 & 9 & 12 & 16 & 42 \\
\hline
\end{tabular}

\section{CXCR4 and CXCL12 mRNA expression in cancerous and normal bladder tissues}

Semiquantitative mRNA analysis revealed that CXCR4 and CXCL12 mRNAs were expressed in varying amounts in cancerous tissues, but not in normal tissues (Figure 2). With regard to different stages of bladder cancer, CXCR4 and CXCL12 mRNA expressions were the highest in $T_{2} T_{3}$ stage (15 cases), followed by $T_{1}$ stage (11 cases), and $T_{a}$ stage (16 cases), with significant intergroup differences $(F=56.642,67.928, \mathrm{P}<0.01)$.

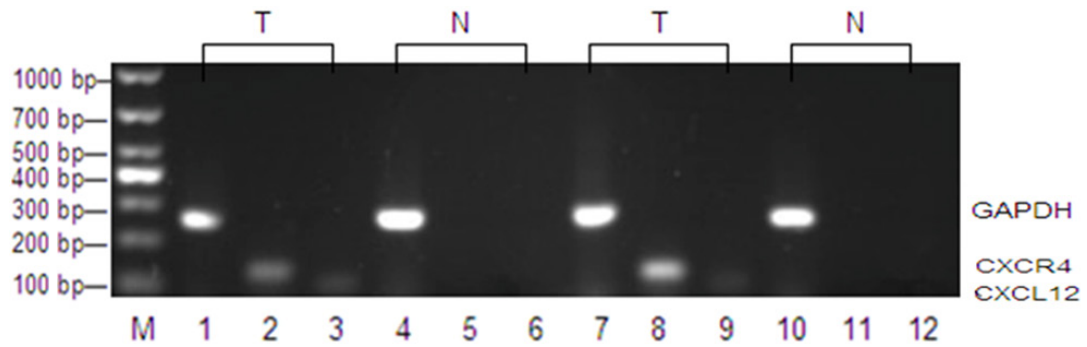

Figure 2. Semiquantitative mRNA analysis (GAPDH, 258 bp; CXCR4, 126 bp; CXCL12, 104 bp). The expression of CXCR4 and CXCL12 mRNAs are seen in bladder tumor tissues, but not in normal bladder tissues. Lanes 4, 5, 6, 11, 12, and 13 are normal (N) bladder tissues, and lanes 1, 2, 3, 7, 8, and 9 are bladder tumor $(\mathrm{T})$ tissues with different degrees of malignancy.

\section{CXCR4 and CXCL12 protein levels in cancerous and normal bladder tissues}

Western blot analysis revealed that CXCR4 and CXCL12 proteins were expressed in varying amounts in cancerous tissues, but not in normal tissues (Figure 3). With regard to different stages of bladder tumor, CXCR4 and CXCL12 protein expressions were the highest in $\mathrm{T}_{2} \mathrm{~T}_{3}$ stage (15 cases), followed by $T_{1}$ stage (11 cases) and $T_{a}$ stage (16 cases), with significant intergroup differences $(F=59.453,61.732, \mathrm{P}=0.000,0.000, \mathrm{P}<0.01)$.

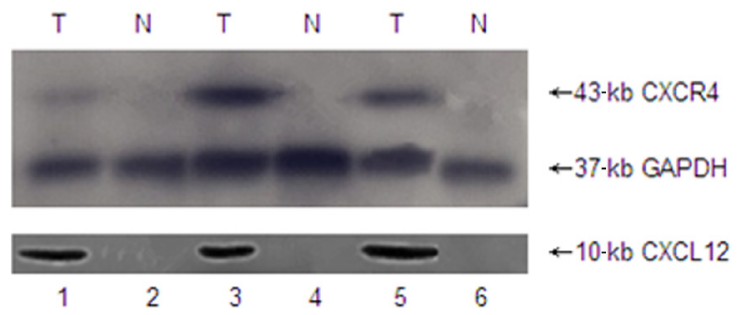

Figure 3. Western blot analysis. The expressions of CXCR4 and CXCL12 proteins are seen in bladder tumor (T) tissues, but not in normal ( $\mathrm{N}$ ) bladder tissues. Lanes 2, 4, and 6 are normal bladder tissues, and lanes 1, 3, and 5 are bladder cancer tissues with different degrees of malignancy. 


\section{DISCUSSION}

Chemokines (relative molecular weight 7-14 kDa) act as chemoattractants for immune cell chemotaxis, such as neutrophils and lymphocytes, and can be produced by various cells. According to the number and position of intramolecular specific cysteines, chemokines can be divided into four sub-families: CXC, CC, C, and CX3C, and likewise, their corresponding receptors can be divided into four types: CXCR, CCR, CR, and CX3CR (Horuk, 2001). CXCR4 is a seven-transmembrane G-protein-coupled receptor that contains 352 highly conserved amino acids. CXCR4 is also expressed in several types of tumor cells. Its specific ligand CXCL12, also known as stromal cellderived factor 1 (SDF-1) or pre-B cell stimulating factor (PBSF), belongs to the CXC chemokine family. CXCL12 and CXCR4 show high affinity for one another and can be specifically combined to form the CXCL12/CXCR4 axis, which is the molecular basis of their biological functions and is closely related to the invasion and metastasis of malignant tumors (Roland et al., 2003; Yasumoto et al., 2011). The CXCL12/CXCR4 axis functions as a regulatory element for leukergy, and can have many effects on the extracellular matrix and on tumor cells (Domanska et al., 2013). The activation of chemokine signals can result in various intracellular changes, including the activation of RAS/MAPK (mitogen activated protein kinase) signal transduction pathways, the polymerization of intracellular actins, the rearrangement of the cytoskeleton, the formation of pseudopods, the movements of cells, and consequently can increase tumor cell invasiveness (Luo et al., 2006).

There have been many reports on CXCR4 expression in cells from various tumors, such as those from breast, lung, pancreas, colon, ovary, and prostate cancers (Balkwill, 2004; Toyozawa et al., 2012; Dai et al., 2013; Zhang et al., 2013), as well as reports on CXCL12 expression in tumor cells, such as those from the primary foci in lymph and neuroglia tumors, and from cancers of the ovary and pancreas (Lira and Furtado, 2012). Studies on bladder cancer have shown that CXCR4 was expressed in bladder tumor cells, but not in normal bladder mucosa, and was more highly expressed in infiltrative tumors than in superficial tumors (Retz et al., 2005). Furthermore, CXCR4 mRNA expression was upregulated in infiltrative and advanced bladder cancer tissues, whereas it was low in superficial bladder cancer (Balkwill, 2004). However, no previous report has definitively confirmed the expression of CXCL12 in bladder tumor tissues, or the correlation between the expression levels of CXCR4 and CXCL12.

In the current study, IHC analysis revealed that CXCR4 and CXCL12 were more expressed in tumor-invaded bladder tissues than in normal tissues, indicating that CXCR4 and its ligand CXCL12 are associated with the occurrence of bladder cancer. Association analyses of the degree of CXCR4 and CXCL12 expressions in bladder tumor tissues to clinical pathological features revealed that CXCR4 and CXCL12 expressions were associated with the invasive depth and differentiation degree of bladder tumors, but not to the gender or age of patients, indicating the important roles of CXCR4 and CXCL12 in the development, invasion, and metastasis of bladder cancer. Further correlation analysis revealed that the expression of CXCR4 was significantly positively correlated with CXCL12 expression, indicating a synergistic effect and potential interdependence between CXCR4 and CXCL12 in bladder cancer.

RT-PCR results show that CXCR4 and CXCL12 mRNAs were not expressed in normal bladder tissues. The results further demonstrate that CXCR4 and CXCL12 mRNAs were expressed in all bladder cancer tumor tissues, the expressions were lower in early-stage cancer tissues and that they were increased with increased invasive depth. These results suggest that during the progression and invasion of bladder cancer, the expression of CXCR4 and CXCL12 mRNAs 
are increased, which may also indicate that bladder cancer cells with high CXCR4 and CXCL12 expressions are more invasive.

All results herein show that the high CXCR4 and CXCL12 expressions are closely related to the development, invasion, and metastasis of bladder cancer, and suggest that bladder tumor cells with high CXCR4 and CXCL12 expressions are highly invasive. Therefore, the invasive depth, degree of metastasis, and prognosis may be evaluated by CXCR4 and CXCL12 expressions in bladder cancer tissues. In vitro studies showed that blocking CXCR4 expression could impact the functions of the CXCL12/CXCR4 axis and repress the growth of bladder tumor cells (Wang et al., 2011). In vivo studies also showed that blocking CXCR4 expression could repress the growth of bladder tumor cells, which provides a potential molecular target for prevention and treatment of bladder cancer.

\section{Conflicts of interest}

The authors declare no conflict of interest.

\section{ACKNOWLEDGMENTS}

Research supported by the National Natural Science Foundation of China (\#81460385), the Yunnan Provincial Department of Education Fund (\#2014Z072) and the Joint Project of Science and Technology, Department of Yunnan and Kunming Medical University (\#2014FZ031).

\section{REFERENCES}

Balkwill F (2004). The significance of cancer cell expression of the chemokine receptor CXCR4. Semin. Cancer Biol. 14: 171179.

Baron V, Adamson ED, Calogero A, Ragona G, et al. (2006). The transcription factor Egr1 is a direct regulator of multiple tumor suppressors including TGFbeta1, PTEN, p53, and fibronectin. Cancer Gene Ther. 13: 115-124.

Calogero A, Arcella A, De Gregorio G, Porcellini A, et al. (2001). The early growth response gene EGR-1 behaves as a suppressor gene that is down-regulated independent of ARF/Mdm2 but not p53 alterations in fresh human gliomas. Clin. Cancer Res. 7: 2788-2796.

Dai X, Mao Z, Huang J, Xie S, et al. (2013). The CXCL12/CXCR4 autocrine loop increases the metastatic potential of non-small cell lung cancer in vitro. Oncol. Lett. 5: 277-282.

Dobner BC, Riechardt Al, Joussen AM, Englert S, et al. (2012). Expression of haematogenous and lymphogenous chemokine receptors and their ligands on uveal melanoma in association with liver metastasis. Acta Ophthalmol. 90: e638-e644.

Domanska UM, Kruizinga RC, Nagengast WB, Timmer-Bosscha H, et al. (2013). A review on CXCR4/CXCL12 axis in oncology: No place to hide. Eur. J. Cancer 49: 219-230.

Horuk R (2001). Chemokine receptors. Cytokine Growth Factor Rev 12: 313-335.

Lira SA and Furtado GC (2012). The biology of chemokines and their receptors. Immunol. Res. 54: 111-120.

Luo C, Pan H, Mines M, Watson K, et al. (2006). CXCL12 induces tyrosine phosphorylation of cortactin, which plays a role in CXC chemokine receptor 4-mediated extracellular signal-regulated kinase activation and chemotaxis. J. Biol. Chem. 281: 30081-30093.

Raghavan D (2012). Introduction: bladder cancer. Semin. Oncol. 39: 523.

Retz MM, Sidhu SS, Blaveri E, Kerr SC, et al. (2005). CXCR4 expression reflects tumor progression and regulates motility of bladder cancer cells. Int. J. Cancer 114: 182-189.

Roland J, Murphy BJ, Ahr B, Robert-Hebmann V, et al. (2003). Role of the intracellular domains of CXCR4 in SDF-1-mediated signaling. Blood 101: 399-406.

Toyozawa S, Kaminaka C, Furukawa F, Nakamura Y, et al. (2012). Chemokine receptor CXCR4 is a novel marker for the progression of cutaneous malignant melanomas. Acta Histochem. Cytochem. 45: 293-299.

Wang H, Yang D, Wang K and Wang J (2011). Expression and potential role of chemokine receptor CXCR4 in human bladder carcinoma cell lines with different metastatic ability. Mol. Med. Rep. 4: 525-528. 
Yasumoto K, Yamada T, Kawashima A, Wang W, et al. (2011). The EGFR ligands amphiregulin and heparin-binding egf-like growth factor promote peritoneal carcinomatosis in CXCR4-expressing gastric cancer. Clin. Cancer Res. 17: 3619-3630.

Zhang Y, Guo Q, Zhao H, Zhao D, et al. (2013). Expression of CXCR4 is an independent prognostic factor for overall survival and progression-free survival in patients with myelodysplastic syndrome. Med. Oncol. 30: 341. 